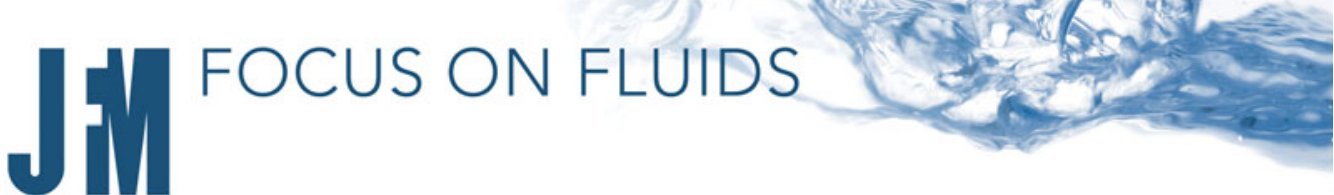

\section{Lagrangian turbulence in the woods}

\author{
Laurent Chevillard $\dagger$ \\ Univ. Lyon, ENS de Lyon, Univ. Claude Bernard, CNRS, Laboratoire de Physique, 46 allée d'Italie, \\ F-69342 Lyon, France
}

(Received 16 February 2021; accepted 22 February 2021)

\begin{abstract}
A recent statistical analysis, proposed by Shnapp (J. Fluid Mech., vol. 913, 2021, R2), of Lagrangian velocity measurements in a wind tunnel in the presence of a canopy (a forest or urban morphology), using three-dimensional particle tracking velocimetry (Shnapp et al., Sci. Rep., vol. 9, issue 1, 2019, pp. 1-13), is a great read. In this strongly anisotropic situation, despite the additional roughness induced by the canopy, it is shown that fluctuations of Lagrangian velocity increments over small time scales display very similar behaviour as those observed in homogeneous and isotropic turbulent flows. This is all the more true when focussing on the non-Gaussian and intermittent nature of these fluctuations. At much larger time scales, of the order and greater than the characteristic turnover time scale of the flow, anisotropies implied by the presence of the canopy are quantified using averages of the fluctuating kinetic energy conditioned upon the direction of Lagrangian velocity with respect to the mean Eulerian flow. Shnapp (2021) evidences that, indeed, the canopy modifies the velocity along the trajectories at large scales, in particular its variance, but leaves unchanged its local regularity, as it is pinpointed by the power-law exponents of the structure functions.
\end{abstract}

Key words: turbulent flows, turbulent boundary layers, intermittency

\section{Introduction}

The Lagrangian investigation of laboratory and numerical turbulent flows has been intensively developed over the last thirty years, as reviewed in Yeung (2002), Toschi \& Bodenschatz (2009) and Pinton \& Sawford (2012), following an intense and vast effort aimed at characterizing with precision the statistical behaviour of the Eulerian velocity field (Frisch 1995). Following velocity along the path of fluid particles is not only important from a fundamental point of view (Monin \& Yaglom 1971; Tennekes \& Lumley 1972 ), it is also an appropriate way to describe the mixing and dispersion properties of emitted tracers in geophysical situations (LaCasce 2008).

Fluctuations of velocity along trajectories were initially observed in direct numerical simulations (DNSs) of the Navier-Stokes equations in controlled situations aimed at

$\dagger$ Email address for correspondence: laurent.chevillard@ens-lyon.fr 


\section{Chevillard}

investigating some aspects of homogeneous and isotropic turbulence (Yeung \& Pope 1989; Chevillard et al. 2003; Biferale et al. 2004; Bentkamp, Lalescu \& Wilczek 2019), and soon after in von Kármán swirling flows (Voth, Satyanarayan \& Bodenschatz 1998; Mordant et al. 2001). Despite the artificial nature of the boundary conditions of DNSs, and the strong anisotropy of the experimental realisation of such flows driven by propellers, a remarkable agreement was observed in the statistical properties of the Lagrangian velocity in both situations (Arneodo et al. 2008). The Johns Hopkins Turbulence Database (Yu et al. 2012) could, for instance, be used to further confirm the picture which follows.

We recall in a few words some of the key ingredients of the phenomenology of homogeneous and isotropic turbulence in the Lagrangian framework, as reviewed by Chevillard et al. (2012). Using the notation of Shnapp (2021), the statistical and multiscale nature of a Lagrangian velocity component $v_{i}(t)$, with $i \in\{1,2,3\}$, is well captured by the following probabilistic ansatz:

$$
\Delta_{\tau} v_{i}=\mathcal{B}\left(\frac{\tau}{T_{L}}\right) \Delta_{T_{L}} v_{i},
$$

where the velocity increment $\Delta_{\tau} v_{i}(t)=v_{i}(t+\tau)-v(t)$ is introduced. Equation (1.1) relates an equality in probability law between the random variable $\Delta_{\tau} v_{i}$, made up of the instances of the increments along the trajectories at a given time scale $\tau$, and its instances $\Delta_{T_{L}} v_{i}$, at the large integral time scale of the flow $T_{L}$ at which velocity decorrelates, weighted by a random scale-dependent multiplier $\mathcal{B}$. At large scales $\tau \gg T_{L}, \mathcal{B}$ tends to the deterministic value 1 , meaning that the increment is statistically equal to $\Delta_{T_{L}} v_{i}$, usually taken to be a Gaussian random variable of zero average and of variance $2\left\langle v_{i}^{2}\right\rangle$, as dictated by observations. In the inertial range $\tau_{\eta} \ll \tau \ll T_{L}$, where $\tau_{\eta}$ is the Kolmogorov dissipative time scale, $\mathcal{B}$ fluctuates in the same way as $\left(\tau / T_{L}\right)^{h}$, the randomness being encoded in the exponent $h$. Dimensional arguments, mostly based on the irrelevance of viscosity at these scales (Tennekes \& Lumley 1972), suggest that $\langle h\rangle \approx 1 / 2$, at any scale $\tau$. Using the language of the multifractal formalism (Frisch 1995), in this statistically averaged sense, we can say that the Lagrangian velocity shares the same local regularity as that of the Brownian motion. Further analyses of experimental and numerical data (Chevillard et al. 2003; Arneodo et al. 2008) indicate that, indeed, $h$ fluctuates around its mean value, independently of both the Reynolds number and the geometry of the flow, which is known as intermittency. The level of Lagrangian intermittency is observed in the right proportion compared to that measured in the Eulerian framework, consistently with the elegant theory of Borgas (1993).

\section{Overview}

Much more could be said on the statistical behaviour of the multiplier $\mathcal{B}$ entering in (1.1), in particular on the rich and predictive physics that has been developed to include the differential action of viscosity at small scales $\tau \ll \tau_{\eta}$, where fluctuations of the velocity increment are similar to those of acceleration. Let us keep in mind that this ansatz is well posed and closed from a probabilistic point of view if we furthermore assume that $h$ and $\Delta_{T_{L}} v_{i}$ are statistically independent. It is then possible to derive explicit predictions for the probability density function (p.d.f.) of $\Delta_{\tau} v_{i}$ and its moments (i.e. the structure functions), at any scale $\tau$, for a given Reynolds number and a prescribed level of intermittency.

The novelty of the analysis of Shnapp (2021) is to show that this aforementioned Lagrangian phenomenology, initially designed for isotropic turbulent flows, gives a fair account of the fluctuations of velocity along the trajectories obtained in his wind tunnel 


\section{Lagrangian turbulence in the woods}

(Shnapp et al. 2019). Despite the presence of a strong mean flow, and a model canopy laid out on the bottom of the tunnel, Shnapp (2021) evidences that the subset of particles flying just above this rough surface exhibits a statistical behaviour in quantitative agreement with the theoretical predictions for the velocity increment p.d.f.s and moments obtained within this formalism, with furthermore the same level of intermittency. Noticing that the characteristic height of the canopy is of the order of $U_{\infty} T_{L}$, where $U_{\infty}$ is the wind mean velocity, this important observation further illustrates that Lagrangian fluctuations at small scales are universal and decoupled from the large scale flow. Nonetheless, signatures of the anisotropic nature of the canopy are evidenced when comparing the variance and correlation time scales of the different components $v_{i}$, which impact $\Delta_{T_{L}} v_{i}$, changing weakly the distribution of $\mathcal{B}$, similarly to what was observed by Ouellette et al. (2006) and Huck, Machicoane \& Volk (2019).

To characterize more precisely the anisotropic nature of the large scale flow from a Lagrangian perspective, Shnapp (2021) decomposes the set of the trajectories according to four quadrants which represent different directions of Lagrangian velocity with respect to the mean Eulerian flow. This original method of classification allows him to analyse the distribution of kinetic energy depending of the amplitude of the streamwise component, and makes some connections with the fluctuating nature of the drag induced by the canopy.

\section{Future}

The results of Shnapp (2021) remarkably show that Lagrangian tracking experiments are an original and fertile characterization of realistic turbulent flows, such as wind tunnels (Ayyalasomayajula et al. 2006; Shnapp et al. 2019), jets (Poulain et al. 2004; Viggiano et al. 2021) and channel flows (Stelzenmuller et al. 2017). These newly developed techniques shed new light on Eulerian measurements and simulations of modelled canopies (Bai, Katz \& Meneveau 2015; Glick et al. 2020), and their consequences for the intermittency phenomenon (Katul et al. 2006; Dupont et al. 2020).

From a theoretical perspective, the Lagrangian framework naturally calls for the stochastic modelling of the trajectories using random walks, as they were developed for isotropic flows (Sawford 1991; Pope 2002; Viggiano et al. 2020). In the spirit of recent propositions made by Innocenti et al. (2020) and Shnapp et al. (2020), generalizing these approaches to anisotropic situations sounds like a fantastic perspective.

Declaration of interests. The author reports no conflict of interest.

\section{REFERENCES}

ARneOdo, A., et al. 2008 Universal intermittent properties of particle trajectories in highly turbulent flows. Phys. Rev. Lett. 100, 254504.

Ayyalasomayajula, S., Gylfason, A., Collins, L.R., Bodenschatz, E. \& Warhaft, Z. 2006 Lagrangian measurements of inertial particle accelerations in grid generated wind tunnel turbulence. Phys. Rev. Lett. 97 (14), 144507.

Bai, K., KatZ, J. \& Meneveau, C. 2015 Turbulent flow structure inside a canopy with complex multi-scale elements. Boundary-Layer Meteorol. 155 (3), 435-457.

BentKamp, L., Lalescu, C.C. \& WilczeK, M. 2019 Persistent accelerations disentangle Lagrangian turbulence. Nat. Commun. 10, 3550.

Biferale, L., Boffetta, G., Celani, A., Devenish, B.J., Lanotte, A. \& Toschi, F. 2004 Multifractal statistics of Lagrangian velocity and acceleration in turbulence. Phys. Rev. Lett. 93 (6), 064502.

Borgas, M.S. 1993 The multifractal Lagrangian nature of turbulence. Phil. Trans. R. Soc. Lond. A 342 , 379.

Chevillard, L., Castaing, B., Arneodo, A., Lévêque, E., Pinton, J.-F. \& Roux, S. 2012 A phenomenological theory of Eulerian and Lagrangian velocity fluctuations in turbulent flows. C.R. Phys. 13,899 . 


\section{Chevillard}

Chevillard, L., Roux, S.G., Lévêque, E., Mordant, N., Pinton, J.-F. \& Arneodo, A. 2003 Lagrangian velocity statistics in turbulent flows: effects of dissipation. Phys. Rev. Lett. 91, 214502.

Dupont, S., Argoul, F., Gerasimova-Chechinana, E., Irvine, M.R. \& Arneodo, A. 2020 Experimental evidence of a phase transition in the multifractal spectra of turbulent temperature fluctuations at a forest canopy top. J. Fluid Mech. 896, A15.

FRISCH, U. 1995 Turbulence, The Legacy of A.N. Kolmogorov. Cambridge University Press.

Glick, A., Ali, N., Bossuyt, J., Calaf, M. \& Cal, R.B. 2020 Utility-scale solar PV performance enhancements through system-level modifications. Sci. Rep. 10 (1), 1-9.

Huck, P.D., MachicoAne, N. \& VOLK, R. 2019 Lagrangian acceleration timescales in anisotropic turbulence. Phys. Rev. Fluids 4 (6), 064606.

Innocenti, A., Mordant, N., Stelzenmuller, N. \& Chibbaro, S. 2020 Lagrangian stochastic modelling of acceleration in turbulent wall-bounded flows. J. Fluid Mech. 892, A38.

Katul, G., Porporato, A., Cava, D. \& Siqueira, M. 2006 An analysis of intermittency, scaling, and surface renewal in atmospheric surface layer turbulence. Physica D 215 (2), 117-126.

LACASCE, J.H. 2008 Lagrangian statistics from oceanic and atmospheric observations. In Transport and Mixing in Geophysical Flows, Lecture Notes in Physics (ed. J.B. Weiss \& A. Provenzale), pp. 165-218. Springer.

Monin, A.S. \& Yaglom, A.M. 1971 Statistical Fluid Mechanics, vols. 1 \& 2. MIT Press.

Mordant, N., Metz, P., Michel, O. \& Pinton, J.-F. 2001 Measurement of Lagrangian velocity in fully developed turbulence. Phys. Rev. Lett. 87 (21), 214501.

Ouellette, N.T., Xu, H., Bourgoin, M. \& Bodenschatz, E. 2006 Small-scale anisotropy in Lagrangian turbulence. New J. Phys. 8 (6), 102.

Pinton, J.-F. \& SAWford, B.L. 2012 A Lagrangian view of turbulent dispersion and mixing. In Ten Chapters in Turbulence (ed. P.A. Davidson, Y. Kaneda \& K.R. Sreenivasan), pp. 132-175. Cambridge University Press.

Pope, S.B 2002 A stochastic Lagrangian model for acceleration in turbulent flows. Phys. Fluids 14 (7), 2360-2375.

Poulain, C., Mazellier, N., Gervais, P., Gagne, Y. \& Baudet, C. 2004 Spectral vorticity and Lagrangian velocity measurements in turbulent jets. Flow Turbul. Combust. 72 (2), 245-271.

SAWFORD, B.L. 1991 Reynolds number effects in Lagrangian stochastic models of turbulent dispersion. Phys. Fluids A 3 (6), 1577-1586.

SHNAPP, R. 2021 On small-scale and large-scale intermittency of Lagrangian statistics in canopy flow. J. Fluid Mech. 913, R2.

Shnapp, R., Bohbot-Raviv, Y., Liberzon, A. \& Fattal, E. 2020 Turbulence-obstacle interactions in the Lagrangian framework: applications for stochastic modeling in canopy flows. Phys. Rev. Fluids 5 (9), 094601.

Shnapp, R., Shapira, E., Peri, D., Bohbot-Raviv, Y., Fattal, E. \& Liberzon, A. 2019 Extended 3D-PTV for direct measurements of Lagrangian statistics of canopy turbulence in a wind tunnel. Sci. Rep. $9(1), 1-13$.

Stelzenmuller, N., Polanco, J.I., Vignal, L., Vinkovic, I. \& Mordant, N. 2017 Lagrangian acceleration statistics in a turbulent channel flow. Phys. Rev. Fluids 2 (5), 054602.

Tennekes, H. \& Lumley, J.L. 1972 A First Course in Turbulence. MIT Press.

Toschi, F. \& Bodenschatz, E. 2009 Lagrangian properties of particles in turbulence. Annu. Rev. Fluid Mech. 41, 375.

Viggiano, B., Basset, T., Solovitz, S., Barois, T., Gibert, M., Mordant, N., Chevillard, L., VolK, R., Bourgoin, M. \& CAL, R.B. 2021 Lagrangian diffusion properties of a free shear turbulent jet. arXiv:2102.08333.

Viggiano, B., Friedrich, J., Volk, R., Bourgoin, M., CAL, R.B. \& Chevillard, L. 2020 Modelling Lagrangian velocity and acceleration in turbulent flows as infinitely differentiable stochastic processes. J. Fluid Mech. 900, A27.

Voth, G.A., Satyanarayan, K. \& Bodenschatz, E. 1998 Lagrangian acceleration measurements at large Reynolds numbers. Phys. Fluids 10, 2268.

Yeung, P.K. 2002 Lagrangian investigations of turbulence. Annu. Rev. Fluid Mech. 34 (1), 115-142.

YeunG, P.K. \& POPE, S.B. 1989 Lagrangian statistics from direct numerical simulations of isotropic turbulence. J. Fluid Mech. 207, 531.

Yu, H., Kanov, K., Perlman, E., Graham, J., Frederix, E., Burns, R., Szalay, A., Eyink, G. \& Meneveau, C. 2012 Studying Lagrangian dynamics of turbulence using on-demand fluid particle tracking in a public turbulence database. J. Turbul. 13, N12. 